(RESEARCH ARTICLE)

\title{
Prevalence of the common intestinal parasitic infections and predisposing factors among the asymptomatic primary school children in Finoteselam town, West Gojam Zone, Amhara region (Ethiopia).
}

\author{
Maru Wassie Yenene * and Abayneh Unasho Gandile \\ College of Natural Sciences, Department of Biology, Arba Minch University, Ethiopia.
}

Publication history: Received on 18 June 2020; revised on 06 September 2020; accepted on 09 September 2020

Article DOI: https://doi.org/10.30574/wjarr.2020.8.2.0210

\begin{abstract}
Background: Intestinal parasitic infections (IPIs) have been still quite common in developing countries including Ethiopia, particularly in children with low socio-economic and poor living conditions. Previous studies in Ethiopia have shown high burden of intestinal parasitic infections among the primary school children. The effective prevention and control of parasitic infections require research work based on the identification of intestinal parasites including associated predisposing factors. Objective: The aim of the present study was to investigate the prevalence of common intestinal parasitic infections and predisposing factors among the asymptomatic primary school children. Methods: A school based cross- sectional study was conducted from September, 2019- January, 2020 and a total of 422 asymptomatic primary school children were involved in the study. Specimens of the stool samples were examined using the standard parasite and ova method. Direct microscopy using saline and iodine wet mount preparation was used to examine the presence of eggs and larvae of helminthes and trophozoites and cysts of protozoan parasites and formalin ether concentration method was used for quality assurance. Structured questionnaires were also used to collect quantitative data on socio-demographic characteristics and predisposing factors. Analysis of the data was made manually using the thematic framework method including descriptive statistics. Results: Among the 422 students (aged 5-14 years) participated in the study, 245(58\%) were positive for one or more intestinal parasites. Of the total infected participants, $192(45.5 \%)$ were single infections and 53(12.6\%) were double infections identified. Seven types of intestinal parasitic infections were identified in the present study. The most prevalent parasite was Giardia lamblia 95(22.5\%) followed by hook worm 58(13.7\%). Age groups 5-9 years old were more infected, $173(70.6 \%)$ than age groups 10-14 years old 72(29.4\%). This result also showed parasitic infections were associated with predisposing factors related to infectious cycles. Concluding Remarks and Recommendations: The present study revealed that there was a high prevalence of intestinal parasitic infection among students in the targeted primary schools. Therefore, all stakeholders should give attention to raise awareness about the prevention and control mechanisms of intestinal parasitic infections. Moreover, the health services of the local government should develop a habit of mass public health education and deworming intervention to improve the level of parasitic infection and environmental hygiene in the study area.
\end{abstract}

Keywords: Asymptomatic infection; Intestinal parasites; Parasitosis; Predisposing factors; Prevalence; School children

\section{Introduction}

\subsection{Background of the Study}

Intestinal Parasitic Infections (IPIs) are among the most prevalent diseases in the world, particularly in developing countries with low socio-economic and poor living conditions [1]. It has been estimated to affect 3.5 billion people globally and 450 million are thought to be ill as a result of such infections, with an estimated 200,000 deaths occurring

\footnotetext{
* Corresponding author: Maru Wassie Yenene

College of Natural Sciences, Department of Biology, Arba Minch University, Ethiopia.
} 
annually, and the majority being children [2,3]. The problem is more serious in Sub-Saharan Africa, Asia and Latin America associated with inadequate water supply, lack of environmental sanitation, fast population growth, and other economic and social problems[4].

The major causes of intestinal parasitic infections of global public health concern are the species of protozoan and helminthes $[2,5]$. The estimates indicate that more than 2 billion people are infected with soil transmitted parasites and $25 \%$ of humans are infected with Ascaris lumbricoides, while Necator americanus and Ancylostoma duodenale (hookworms) infect over a billion people worldwide[6,7,8]. Among the intestinal protozoan parasites, Giardia lamblia and Entameoba histolytica have been considered to be two of the most commonly causative agents of persistent and acute diarrhea in children [9,10]. It is estimated that $10 \%$ of the world's population is infected with E. histolytica resulting in 40-100 thousand deaths annually with the highest prevalence in developing countries among young children [11].About 20-30\% prevalence of the global burden of G. lamblia was reported in developing countries, especially in children $[12,13,14]$.

Furthermore, WHO [15] report indicates that more than 280 million children who live in 42 countries of the African Regions are in need of deworming ( $=30 \%$ of the global total). Furthermore, another study which was done in children of aged 17 years and below from East African countries shows 50\% prevalence rate and all infected children were asymptomatic during diagnosis [16]. In another study in Nigeria, 28\% of studied children were infected with one or more intestinal parasites [17].

Study result show in Ethiopia, the prevalence of intestinal parasites among the school children was $34.2 \%$ [18].Moreover, several studies indicate that the prevalence of helminthic infections were high in the lower altitudes of Ethiopia [19] and A. lumbricoides, was the most prevalent intestinal parasites in different communities usually occurring together with Trichuris trichiura and hookworm [20].

Studies show, factors like use of human excreta as fertilizers, lack of shoes wearing, low household income, limited access to clean drinking water supply, poor sanitation and lack of toilet facilities; eating unwashed fruits and raw vegetables, untrimmed and unclean finger nails, and inappropriate disposal of wastes are predisposing factors of intestinal parasitic infections(IPIs) in different countries including Ethiopia[21,19,22,6].Moreover ,lack of lack of awareness about mode of transmission of parasitic infections increases the risk of parasitic infections[8, 23]..Moreover epidemiological surveys have revealed that, poor sanitation and inappropriate environmental conditions coupled with indiscriminate defecation and contamination of water bodies are the most important predisposing factors to intestinal infections $[24,25]$. Other practices such as over-crowding, improper hand washing, lack of personal hygiene may also contribute to the intestinal parasitic infection [26, 27].In consideration of predisposing factors of parasitic infection, Ethiopia has one of the lowest qualities of drinking water supply and latrine coverage in the world [28] and as comparison by the year 2000, Ethiopia had only $12 \%$ latrine coverage while Kenya had 87\% [29, 19]. Furthermore, like other sub-Saharan African countries, diarrheal infection is a leading cause of morbidity and mortality among young children in Ethiopia, where the total number of annual deaths due to diarrheal illness has been estimated more than 73,000 children [30].

Intestinal infections are mainly transmitted via ingestion of water, soil or food being contaminated by feces containing the cysts of protozoan parasites, or eggs/larvae of helminthes [1, 31].

Furthermore, intestinal parasitic infections have been linked with an increased risk for nutritional anemia, proteinenergy malnutrition and growth deficits in children [32,33,34] and defective school attendance of school aged children (Connolly and Kvalbvig,1992 cited in [35].

A study on the prevalence of intestinal parasitic infection and predisposing factors is the prerequisite not only to facilitate ground for the formation of appropriate control strategies but also to assess health status of the communities in developing countries including Ethiopia [29, 36, 37].Moreover, the study results and findings may increase community awareness and help to design effective control strategies including the research work on IPIs [37,38,39]. However, the prevalence of intestinal parasitic infections and associated risk factors among the asymptomatic school children are not well known in general and in the study area in particular. Thus, the aim of the present study was to assess the current epidemiological prevalence of intestinal parasitic infections and the associated risk factors among the asymptomatic primary school children in the study area 


\section{Material and methods}

\subsection{Description of the Study Area}

Ethiopia is one of the sub- Saharan African countries located in the horn of Africa. It is extremely ethnically diverse country inhabited by more than 80 ethnic groups of which Amhara ethnic group is inhabited in Amhara regional state. Finote selam is the zonal capital of west Gojjam zone which is located in southern part of Amhara national regional state (Figure,1). The town is found 387kms west of Addis Ababa which is geographically, located at 10'41'34" North of latitude and 370 16' 84" East of longitude as reported by Finote Salam City Administration and the town has a total area of 42.65 square kilometers and has a population of 46,539 (21,301 males and 25,238 females). The population has (94.98 \%) Orthodox, 4\% Muslim and 1.02\% Protestant religions [40]. According to Finote selam city administration Office [40], the town annual average temperature is about 23 degree centigrade and elevation of 1860 above sea level. The annual rain fall is around $1250 \mathrm{~mm} /$ year. Finote selam town has five kebeles, 2 private kindergarten (KG) schools (1-3), 8 governmental primary schools, 3 secondary schools (2 from grade 9-10 and 1 from grade 11-12), one health center, one hospital and 7 private clinics.

However, school based infection of intestinal parasites was not studied well and documented in the targeted study area.

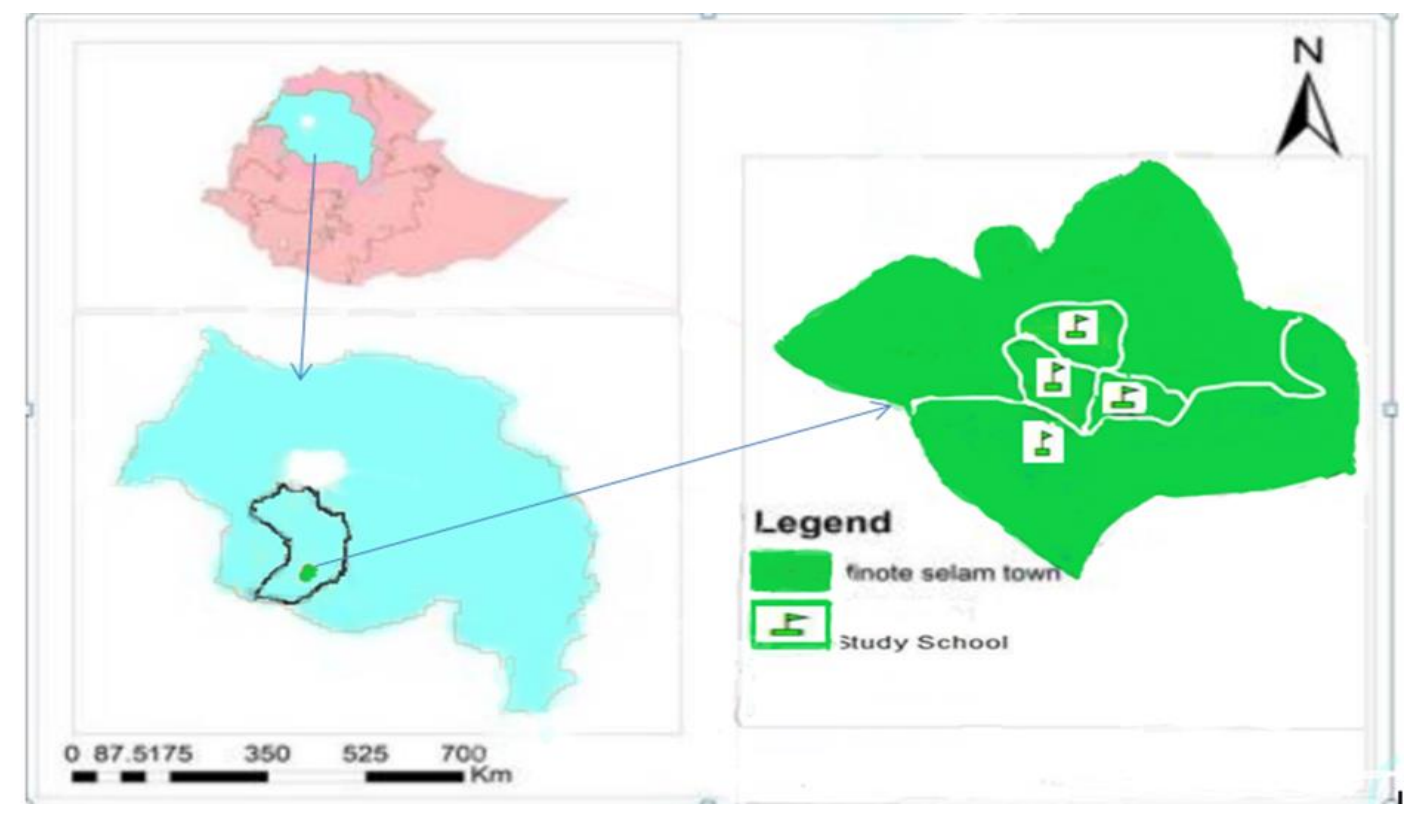

Figure 1 Map of Ethiopia Showing Amhara Region and Fitnoteselam town [41] located in Ethiopia

\subsection{Study Design}

Experimental design including the school based cross- sectional study was conducted from September, 2019- January, 2020 at the randomly selected four primary schools including (Edigetber, Bakel, Wojet and Firin) at the Finote selam town west Gojjam zone Amhara region. Finote selam town was selected for the study because; previously the prevalence of intestinal parasitic infections was not reported in this area. Parasitological techniques involving stool examination of the direct microscopy was conducted at the selected primary schools and concentration method of stool examination was done at Finoteselam hospital. Stool specimens were examined by the skilled laboratory technologist of Finoteselam hospital and all the data analysis and interpretations were done by the investigators.

\subsection{Study Participants and Techniques of Sampling}

The technique of sampling used, was random sampling method and the study participants included were primary school asymptomatic children at Finote selem town. From the total 4,708 four primary school children, 422 students were randomly selected and participated in the study. The technique of sampling to select the study participants of the asymptomatic school children included eligibility criteria having both exclusion and inclusion criteria i.e. 
Inclusion criteria included:

- $\quad$ All the students who were randomly selected from the study population.

Exclusion criteria included:

- $\quad$ Students who were absent from their class session due to illness or who did not show willingness to participate in the study.

- Students who took treatment /anti parasitic drug for any intestinal parasitic infection within the three months prior to the study.

- Students who showed clinical symptoms such as diarrhea, abdominal discomfort, and vomiting during the data collection were intentionally excluded from the study participants.

\subsection{Sample Size Determination}

\subsubsection{Sample Size Determination}

The sample size was computed on the formula proposed for a single population proportion [42]. Therefore, the sample size was calculated using statistical formula considering $95 \%$ confidence interval and $5 \%$ marginal error, i.e. $\mathrm{n}=$ $\frac{(z \alpha / 2)^{2} p(1-p)}{d 2}$

$\mathrm{n}=\frac{(1.96)^{2} 0.5(1-0.5)}{(0.0025)}=384.16$

Where:

- $\mathrm{n}=$ number of sample size

- $\mathrm{d}=$ marginal error between the samples and populations $(0.05)$

- $\mathrm{z} \alpha / 2=$ critical value for normal distribution at 95\% certainty/confidence interval(1.96)

- Expected prevalence of infection (p) = for estimating the sample size, $50 \%$ of the prevalence was taken, because there was no data recorded for the prevalence of common intestinal parasitic infection in the study area.

- Non- response rate was considered and adjusted adding $10 \%$ contingency to generate representative sample size $(n=38+384=422)$ in the randomized cross-sectional survey study.

\subsubsection{Sampling Techniques}

Participants were chosen based on the principles of multistage sampling technique (i.e. stratified and systematic random sampling techniques) due to the disproportionate distributions of the students across various grade levels (= grade 1 to 6 primary schools).

Considering the proportional ratio, $\mathrm{n} / \mathrm{N}(422 / \mathrm{N}(\mathrm{N}=$ total number of students from grade 1 to 6$)=\mathrm{x}(\mathrm{x}$ times the total number of students from each grade to gain the sample size $(\mathrm{n}=422)$. Total number of students in four primary schools in the present study was 4708 and the sample size is 422 , so proportional ratio is equal to $422 / 4708=0.0896$; to find the sample size, the total number of students from each primary school was multiplied by the proportional ratio $(=$ 0.0896), (Edigetber $920 \times 0.0896=82$, Wojet $1300 \times 0.0896=117$, Bakel $1053 \times 0.0896=94$ and Firin $1435 \times 0.0896=129$ ).

The sample size was distributed proportionally to each grade level of the randomly selected schools according to the number of students in each grade. Finally, each student involved in the study was stratified into classes and was selected using systematic random sampling technique. Since the sample size was 422 from the total student population /Universe of $\mathrm{N}$, then the sampling interval was 4708/422=11 then, the random number between 1 and 11 selected using simple random (lottery method) and the random number was 3 and then the first unit/number selected from each class was the third role number of the students, listed in the class room roster and the second was $3+11=14$, and the third was $14+11=25$ and so on until the sampling frame was exhaustively used. 


\subsection{Method of Data Collection}

\subsubsection{Questionnaire Survey}

After written consent was obtained from parents (caretakers) of the children, completing, structured questionnaires were used to collect quantitative data on socio-demographic characteristics including age, sex, educational status, family size, family monthly income, occupation, religion, family relationship and associated predisposing factors with intestinal parasitic infection from all selected participants. The questionnaires were administered in English, and then translated into local language (Amharic) to collect the data. Structured questionnaires were distributed to the selected 422 respondents and parents (caretakers) for younger children during collection of stool samples from the study participants at selected primary schools.

\subsubsection{Stool collection}

After written consent was obtained from every participant/ parents or caretakers of the selected children, the investigator with school directors prepared a room for microscopic examination at the school compounds of each primary school. Then, after proper instructions were given, students/caretakers were given labeled clean, wide-mouth containers with tightfitting lids containing a few drops of physiological saline and applicator sticks to bring fresh stool for parasitological examination. 2-4gms of fresh stool were collected from each participant individuals. At the time of collection, all stool specimens were properly labeled with date of sampling, identification number; age and sex were recorded for each participant on a recording format. Standard parasitological techniques were used and stool examination for intestinal parasites was conducted using standard routine intestinal parasite examination.

\subsubsection{Direct Wet Mount and concentration Methods}

A small portion of each of fresh, unpreserved stool samples were emulsified with normal physiological saline $(0.85 \%$ $\mathrm{NaCl}$ solution) being placed on a slide. The suspension on the slide was carefully covered with a cover slip in a way to avoid air bubbles and parasitological examination (=Direct wet mount method) was processed and examined microscopically within 15-20 minutes to observe the presence of trophozoites, cysts, eggs and larva of intestinal parasites under light microscope at $10 \mathrm{X}$ and $40 \mathrm{X}$ magnification power. Weak iodine wet mount was also used to observe nuclear characteristics of common intestinal parasitic infections [43]. After direct wet parasitological examination, 10\% of the total numbers of positive stool samples were preserved in $5 \%$ formalin for protozoan parasites and $10 \%$ formalin for helminthic parasites and transported to Finote selam hospital Laboratory room on the same day to recheck positive stool specimen for quality assurance using standard method of concentration techniques.

\subsection{Method of Data Analysis}

Descriptive statistics including tables, frequencies and proportions were used to give a clear picture of background characteristics such as age, sex, educational background, family size, family monthly income, prevalence of parasitic infections and associated predisposing factors of infections, infection rate and the distribution of the parasites among the asymptomatic primary school children. Data analysis was done manually using quantitative and qualitative research parameters involving thematic framework method including descriptive statistics.

\subsection{Data Quality Control}

To ensure quality of data collection, all the laboratory procedures including collection and handling of specimens were carried out in accordance with the standard protocols [44]. During data collection, all the activities of the work (stool collection, handling and parasite identification) were carefully monitored by the laboratory technologist and the researchers. Furthermore, to control the data quality, $10 \%$ of the total numbers of positive stool sample were preserved in 5\% formalin for protozoan parasite and 10\% formalin for helminthic parasites [45] and were examined in Finote selam hospital laboratory room by the anonymous laboratory technologist using Formal Ether Concentration Method and direct microscopy to recheck the results. There was no discrepancy on the stool examination results between the first positive test and the quality control test. So, the results of the new laboratory examination were used as a quality control.

\subsection{Ethical Consideration}

Prior to research conduction, ethical approval was obtained from the Arba Minch University department of biology and the letter describing the objective of the research was written to Finote selam town administration educational office and permission was obtained from Finote selam town educational authorities and school principals before the actual investigation was began. The objective of the study was explained to school communities, kebele leaders and parents. Written consent was obtained from every participant's parent or caretakers of the selected children before conducting 
the study and the children also gave their assent before specimen collection. Moreover, explanation was given about the procedure of stool examination that it is non-invasive and has no harm to the study participants and the objective of the study result may benefit the study participants as well the community. Privacy and confidentiality of the information was ensured. The ethical considerations were addressed by treating positive children using standard drugs guided by the prescription of the pediatrician and mothers were also told to report to pediatrician if they encounter any side effects/challenges.

\section{Results}

\subsection{Results}

\subsubsection{Socio-Demographic Characteristics of the Study Participants}

.A total of 422 study participant were selected for the diagnosis of intestinal parasites, from which 221 (52.4\%) were females and the remaining 201(47.6\%) were males. Based on the examined stool samples, of the 201 (47.6\%) males enrolled, 123 (29.1\%) were infected, while, of the total 221(52.4) females, 122 (28.9\%) were infected with intestinal parasitic infections in the present study.

The mean age of the study participants was 9 years old with minimum and maximum age groups of 5 and 14 years old respectively. About $243(57.6 \%)$ of the children were in age range of 5-9 years old and $179(42.4 \%)$ of them were within age range of 10-14 years old. Of the student parents/caretakers, 38(9\%) were illiterate, 105(24.9\%) were primary school (grade1-8) and 279(66.1\%) were secondary school and above in their educational status. Furthermore, most of the study participants, $279(66.1 \%)$ came from family sizes $<5$ and $143(33.9 \%)$ came from family sizes $\geq 5$, and $113(26.8 \%)$ have $\leq 3500$ Birr (ETB), 309(73.2\%) have $>3500$ (ETB) monthly income and the result was shown in Table 1.

Table 1 Socio demographic characteristics of the study participants and their families

\begin{tabular}{|c|c|c|c|}
\hline Variables & Categories & Frequency & Percent (\%) \\
\hline Gender of the- & Male & 201 & 47.6 \\
\hline \multirow[t]{2}{*}{ School Children: } & Female & 221 & 52.4 \\
\hline & Total & 422 & 100 \\
\hline \multirow[t]{2}{*}{ Age of children: } & $5-9$ & 243 & 57.6 \\
\hline & $10-14$ & 179 & 42.4 \\
\hline Family & $\begin{array}{l}\geq 5 \\
<5\end{array}$ & $\begin{array}{c}143 \\
279\end{array}$ & $\begin{array}{c}33.9 \\
66.1\end{array}$ \\
\hline Monthly income (Birr): & $\begin{array}{l}\leq 3500 \\
\quad>3500\end{array}$ & $\begin{array}{l}113 \\
309\end{array}$ & $\begin{array}{c}26.8 \\
73.2\end{array}$ \\
\hline \multirow{3}{*}{$\begin{array}{r}\text { Family educational status: } \\
\text { Prin } \\
\text { Secondary }\end{array}$} & Illiterate & 38 & 9 \\
\hline & y school (1-8) & 105 & 24.9 \\
\hline & hool and above & 279 & 66.1 \\
\hline
\end{tabular}

\subsubsection{Prevalence of Intestinal Parasitic Infections among the Asymptomatic Primary School Children}

From the total 422 study participants who were examined for IPIs, 245 (58\%) were positive for one or more intestinal parasites and seven different parasite species were identified. The result indicated different types of intestinal parasites including protozoan and helminthes parasites from the stool sample and the result was shown in Table 2. 
Table 2 Types of parasites and the overall prevalence of intestinal parasitic infections among the study participants in Finote selam town (September, 2019- February, 2020).

\begin{tabular}{|lccc|}
\hline \multicolumn{4}{c}{ Infected students (participants) } \\
Parasite species & Male No. (\%) & Female No. (\%) & Total No. (\%) \\
\hline Protozoa: G.lamblia cyst & $53(12.5)$ & $42(10)$ & $95(22.5)$ \\
E.histolytica/dispar cyst & $11(2.6)$ & $14(3.3)$ & $25(5.9)$ \\
\hline Helminthes: & & & \\
Hookworm egg & $30(7.1)$ & $28(6.6)$ & $58(13.7)$ \\
A.lumbricoides egg & $25(5.9)$ & $23(5.5)$ & $48(11.4)$ \\
T.trichiura egg & $4(1)$ & $9(2)$ & $13(3)$ \\
S.mansoni egg & - & $5(1.2)$ & $1(1.2)$ \\
H.nana egg & - & $1(0.2)$ & $245(58)$ \\
\hline Total & $123(29.1)$ & $122(28.9)$ & $192(45.5)$ \\
\hline Single infection & $91(21.6)$ & $101(21.9)$ & $53(12.6)$ \\
\hline Double infection & $32(7.6)$ & $21(5)$ & \\
\hline
\end{tabular}

In Table 2 above, the prevalence of Giardia lamblia was 95(22.5\%), followed by hookworm 58(13.7\%), Ascaris lumbricoids 48(11.4\%), Entamoeba histolytica/dispar 25(5.9\%), Trichuris trichiura 13(3\%), Schistosoma mansoni 5(1.2\%), and Hymenolepis nana 1(0.2\%). Single and double infections were identified at the rate of 192 (45.5\%), 53 $(12.6 \%)$ respectively. No triple and above infections were found in the present study. The double infections included Hookworm and A. Lumbricoides 18(4.3), Hookworm and E. histolytica 10 (2.4\%), A. Lumbricoides and E. histolytica 9 (2.1\%), Hookworm and G. Lamblia 8(1.9\%), A. lumbricoides and T. trichiura 8(1.9\%).

The number of children with all, positive cases in randomly selected four primary schools was shown in Table 3 below. Accordingly, in Edigetber primary school, out of 82 study participants, 47 (57.3\%) were positive cases followed by 55 (58.1\%) in Bakel, 68(58.1\%), in Wojet, and 75(58.1\%) in Firin primary schools and the prevalence of intestinal parasites in selected schools was shown in Table 3.

Table 3 Prevalence of intestinal parasites among school children in Finoteselam town

\begin{tabular}{|c|c|c|c|c|c|}
\hline \multirow[b]{2}{*}{$\begin{array}{l}\text { Parasites } \\
\text { identified }\end{array}$} & \multicolumn{5}{|c|}{ Schools } \\
\hline & $\begin{array}{l}\text { Edigeber No. } \\
\text { of observed } \\
(\%) n=82\end{array}$ & $\begin{array}{l}\text { Wojet No. of } \\
\text { observed (\%) } \\
n=117\end{array}$ & $\begin{array}{l}\text { Bakel No. of } \\
\text { observed (\%) } \\
\text { n=94 }\end{array}$ & $\begin{array}{l}\text { Firin No. of } \\
\text { observed } \\
(\%) n=129\end{array}$ & $\begin{array}{l}\text { Total No. of } \\
\text { observed } \\
(\%) n=422\end{array}$ \\
\hline $\begin{array}{l}\text { Protozoa: } \\
\text { Gardia lamblia } \\
\text { Entamoeba } \\
\text { histolitica }\end{array}$ & $\begin{array}{l}16(19.5) \\
7(8.5)\end{array}$ & $\begin{array}{l}28(23.9) \\
6(5)\end{array}$ & $\begin{array}{l}20(21.3) \\
2(2.1)\end{array}$ & $\begin{array}{l}31(24) \\
10(7.8)\end{array}$ & $\begin{array}{l}95(22.5) \\
25(5.9)\end{array}$ \\
\hline $\begin{array}{l}\text { Helminthes: } \\
\text { Hookworm } \\
\text { Ascaria } \\
\text { lumbricoides } \\
\text { Trichuris trichiura } \\
\text { Schistosoma } \\
\text { mansoni } \\
\text { Hymenolepis nana }\end{array}$ & $\begin{array}{l}13(15.9) \\
9(11) \\
1(1.2) \\
1(1.2) \\
-\end{array}$ & $\begin{array}{l}15(12.8) \\
11(9.4) \\
4(3.4) \\
3(2.6) \\
1(.9)\end{array}$ & $\begin{array}{l}9(9.6) \\
22(23.4) \\
1(1.1) \\
1(1.1) \\
\end{array}$ & $\begin{array}{l}21(16.3) \\
6(4.7) \\
7(5.4)\end{array}$ & $\begin{array}{l}58(13.7) \\
48(11.4) \\
13(3.1) \\
5(1.2) \\
1(.2)\end{array}$ \\
\hline Over all total & $47(57.3)$ & $68(58.1)$ & $55(58.5)$ & $75(58.2)$ & $245(58)$ \\
\hline
\end{tabular}




\subsubsection{Sex, Age groups and Magnitude (Load) of Individual Parasitic Infection in Asymptomatic school Children}

Based on the examined stool samples, of the 201 (47.6\%) males enrolled, 123 (29.1\%) were infected, and of the total 221(52.4\%) females, 122 (28.9\%) were infected with intestinal parasites. From the total 245 (58\%) infected study participants (school children), 173(41\%) infected students were found among age groups 5-9 years and 72(17\%) infected students were found among age groups 10-14 years (Table 4). Stool examination in the present study showed Giardia lamblia 95(22.5 \%), E. histolitica/dispar 25(5.9\%), Hook worm 58(13.7\%), A. lumbricoides 48(11.4\%), $T$. trichiura 13(3.1\%), S.mansoni 5(1.2\%) and H.nana 1(0.2\%) infections. Stool examined in the present study also showed none of the studied children between 10-14 years of age were infected by H.nana and S. mansoni and the result was shown in Table 4.

Table 4 Age and Sex of children, Types and Magnitude (Load) of Individual Parasitic Infection in asymptomatic school children in different age groups.

\begin{tabular}{|c|c|c|c|c|}
\hline \multirow{2}{*}{\multicolumn{2}{|c|}{$\begin{array}{l}\text { Parasite species } \\
\text { Age groups }\end{array}$}} & \multicolumn{3}{|c|}{ Parasite infected student by age groups } \\
\hline & & 5-9 No (\%) & $10-14$ No. $\%$ & Total $(\%)$ \\
\hline \multirow{2}{*}{\multicolumn{2}{|c|}{ 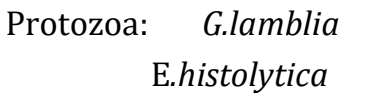 }} & $68(16.1)$ & $27(6.4)$ & $95(22.5)$ \\
\hline & & $14(3.3)$ & $11(2.6)$ & $25(5.9)$ \\
\hline \multirow{3}{*}{\multicolumn{2}{|c|}{$\begin{array}{c}\text { Helminthes: Hookworm } \\
\text { A.lumbricoides } \\
\text { T.trichiura }\end{array}$}} & $37(8.8)$ & $21(5)$ & $58(13.7)$ \\
\hline & & $38(9)$ & $10(2.4)$ & $48(11.4)$ \\
\hline & & $10(2.4)$ & $3(0.7)$ & $13(3.1)$ \\
\hline \multirow{2}{*}{\multicolumn{2}{|c|}{$\begin{array}{l}\text { S.mansoni } \\
\text { H.nana }\end{array}$}} & $5(1.2)$ & - & $5(1.2)$ \\
\hline & & $1(0.2)$ & - & $1(0.2)$ \\
\hline \multicolumn{2}{|r|}{ Total } & $173(41)$ & $72(17)$ & $245(58)$ \\
\hline Sex & Children & \multicolumn{2}{|c|}{ No of positives (\%) } & No of Negatives (\%) \\
\hline Male & 201 & \multicolumn{2}{|c|}{$123(29.1 \%)$} & $78(18.4 \%)$ \\
\hline Female & 221 & \multicolumn{2}{|c|}{$122(28.9 \%)$} & $99(23.4 \%)$ \\
\hline Total: & 422 & \multicolumn{2}{|l|}{$245(58 \%)$} & $177(42 \%)$ \\
\hline
\end{tabular}

\subsubsection{Responses of the participants on the associated predisposing factors}

Responses of the participants were collected on the associated predisposing factors of the parasitic infection from 422 study participants and the results were shown in Table 5 below.

In Table 5, majority of the respondents 225(53.3\%) got drinking water from pipe line and 197(46.7\%) respondents from unprotected well and river while $303(71.8 \%)$ did not dispose household wastes properly, but $119(28.8 \%)$ used to dispose household waste properly. Of the total participants, only 241(57.1\%) used to wash their hands after defecation, but 181(42.9\%) did not wash their hands after defecation. Among the total 422 participants, 192(45.5\%) had frequent river water contact and 230(54.5\%) did not have frequent water contact. Moreover, among the total 422 participants, 236 (55.9\%) used to wash their hands before meal, but, 186 (44.1\%) did not wash their hands before meal. Among the study participants, 157(37.2\%) did not use shoe wearing consistently whilst 265(62.8\%) participants had the habit of shoe wearing consistently. Furthermore, among the study participants, 263(62.1\%), used to eat raw vegetables and unwashed fruits but, 160(37.9\%) did not eat raw vegetables and unwashed fruits. Moreover, 261(61.8\%) participants did not have cleaned and trimmed finger nails while 161(38.2\%) had cleaned and trimmed finger nails during data collection and the result was shown in Table 5. 
Table 5 Responses of the study participants on the associated predisposing factors of parasitic infection among the asymptomatic school children from (September, 2019- January 2020).

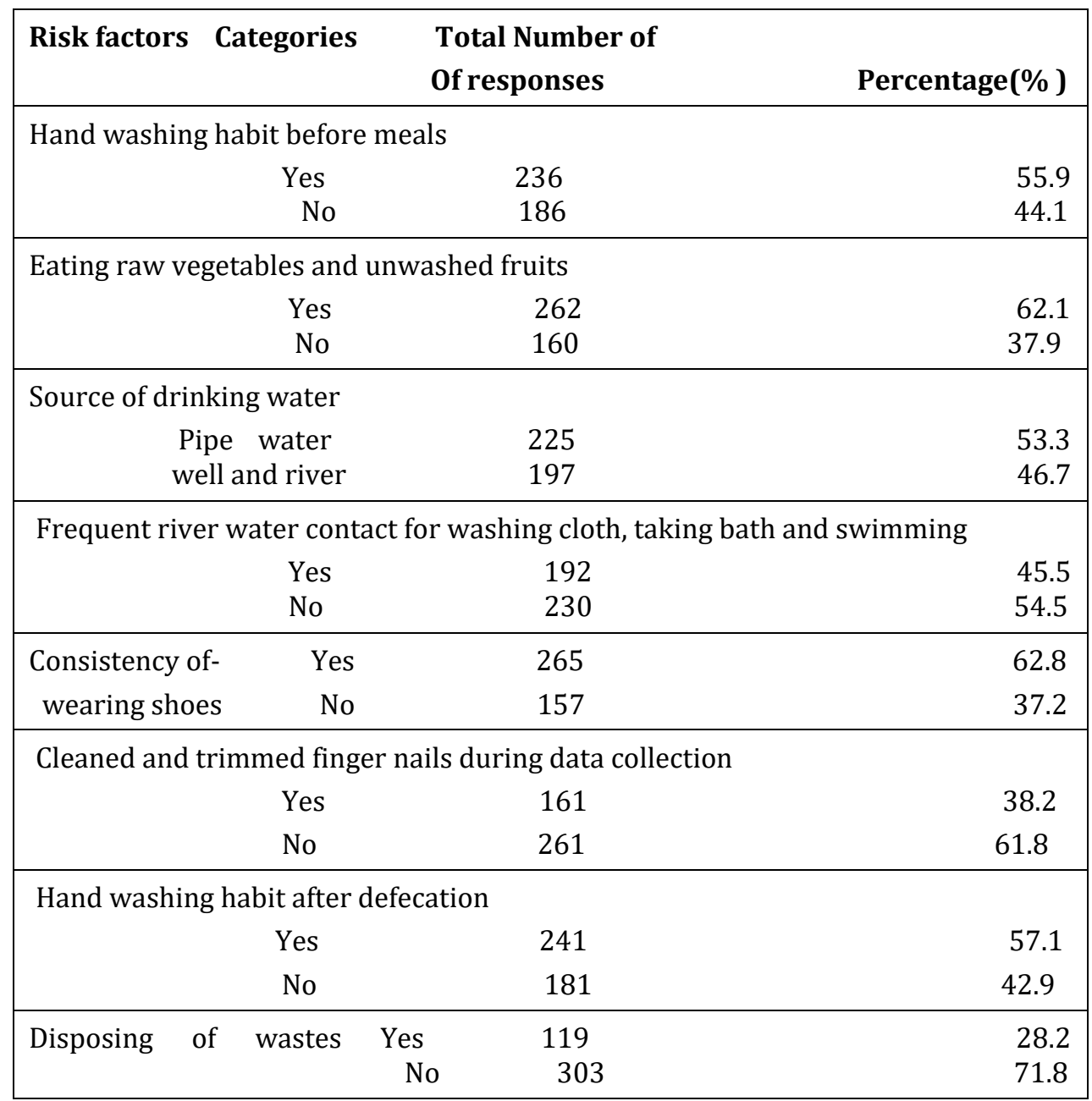

\subsection{Discussion}

Different studies show infection of healthy carriers (=asymptomatic persons), could become a potential source of transmission of variety of infectious agents including intestinal parasites. The present study results among the asymptomatic school children indicated, of the total 422 study participants, the overall prevalence of intestinal parasitic infection was 245(58\%) (Table 4) which was comparable with (54.5\% -66.7\%) study results reported in different parts of Ethiopia including 62.3\% [46,]; 66.7\% [47]; 60.7\% [48]; 54.5\% [49]; 58\%[50] and the study result from Morocco 65\%[51].Moreover, the present finding was higher in comparison with similar study results reported( $21.4 \%-46.6 \%)$ in different parts of Ethiopia and prevalence of intestinal parasites including 27.7\% [52]; 42.1\% [53]; 21.4\% [54]; $39.2 \%$ [35]; 30\%[55] and those of 45.2\% [56]; 17.7\% [57]; 42.3\%[58] reported from Nigeria, Saudia Arabia, and Iran respectively.

In contrast to present findings of intestinal parasitic infections, other study results also show high prevalence of intestinal parasitic infections in different parts of Ethiopia including 77.9\%[59]; 84\% [60]; 70\%[61] and those of 84.7\%[62]; 70\%[63] were also reported from Burkina Faso and Sudan respectively. These reported results differences in the prevalence of intestinal parasitic infection among the different study areas might be associated with environmental differences of parasites distribution, differences in level of environmental sanitation, level of drinking water source, personal hygiene, low level of awareness about the transmission of intestinal parasites and ignorance of health-promotion practices [ $8 ; 23 ; 64]$.

The most prevalent intestinal parasite in the present study was G. lamblia (22.5\%), which is in agreement with $20.1 \%$ $22.8 \%$ reported from school children in different parts of Ethiopia including the prevalence of G.lamblia infection of 
22.8\% [59] and 20.1\%[50]. The present finding also revealed high prevalence of G. lamblia in comparison with similar studies (2.7\% -11.7\% ) reported from different parts of Ethiopia including 6.6\% [23]; 11.7\%[65]; 2.7\%[35] and those of 1.6\% [56] reported from Nigeria. In contrast to the present finding, high prevalence of G. lamblia were also reported in Ethiopia including 41.9\% [53]; 47.7\% [53] and in Rwanda 54.5\% [67] in Burkina Faso 66.5\%[62] and in Yemen $64 \%[39]$. Variations of the results may be due to predisposing factors such unsafe drinking water source, dirt and unclean finger nail, frequent river water contact, open field defecation practices, poor hand washing habit before meal and after defecation, and habit of eating raw vegetables and unwashed fruits $[68,31,69]$.

Hookworm was the second most prevalence parasitic infection (13.7\%) among the asymptomatic students in the present study, which is in agreement with the study results including $11.5 \%-14.1 \%$ reported in similar different studies in Ethiopia including the prevalence of hookworm infection including 11.5\%[66]; 12.2\%[49] and 14.1\%[70].The present study also revealed high prevalence of hook worms infection (13.7\%) in comparison with $2.2 \%-6.7 \%$ reported from similar studies in different parts of Ethiopia including the prevalence of hook worm infection including 6.6\%[46]; $2.2 \%[65] ; 4.9[47] ; 3.9 \%[48]$ and 6.7[53]. However, the prevalence of hookworm infection in the present study was lower than other similar studies reported including 32.5\% in Nigeria [56] and in Ethiopia 33\% [71; 72]. These prevalence variations of hookworm infection among the study results could be difference in the status of environmental sanitation, consistency of shoe wearing habit of the students $[1,31]$.

In the present study, the prevalence of H. nana (0.2\%) was lower than the similar studies reported by other studies in Ethiopia including 1.6\%[23]; 16.7\%[50] and the present study identified lower prevalence of S. mansoni (1.2\%), but higher prevalence than the study conducted in Akaki-kality sub city 0\%, reported by Alemayehu Getachew [23]. These variations in prevalence of parasites among the study results could be due to frequency of river water contact, environmental and personal hygiene and geographical distribution differences of the parasites [73].

G. lamblia, Hook worm, A. lumbricoides, E. histolitica and T. trichiura infections were shown to be the most frequent infections in the age group of 5-9 years (Table 2). However, it showed a decreasing trend in the age groups above 9 years in the present study and this result was supported by other similar studies [23, 72]. The observed higher prevalence in 5-9 year's age group might be due to immature immunity, less awareness of personal hygiene, low level of knowledge about mode of transmission of parasitic infections, increased exposure of children to infective agents with increased mobility and weaning practices, and they usually play on soil, swim/bathe in river water and increased tendency of this age group to eat unwashed fruits, which may be contaminated with infective parasites might have contributed to the higher prevalence of IPIs[74,48,75,50].

The prevalence of double parasitic infections in the present study was about (8.6\%),and this was comparable with other similar studies in Ethiopia including 7\%[52] and lower double infection than other previous studies including 43.9\%[66] and 91.5\%[59] and the present study was also showed higher double infection than other reported results including 3\% [75]. The differences in the prevalence of multiple parasitic infections might be varied in relation to level of environmental contamination, level of awareness about parasitic infection and socioeconomic factors, level of knowledge, attitude and practice towards intestinal parasitic infections [8, 31].

Female participants were slightly greater 221(52.4\%) than males 201(47.6\%), but the prevalence of intestinal parasitic infection in male and female individuals was similar, i.e. $123(29.1 \%)$ and $122(28.9 \%)$, respectively, and there was no great variation of IPIs between male and female study participants (Table 4). It was in agreement with similar studies $[23,73]$. The number of children with all, positive cases in randomly selected four study sites showed almost the same (no great variations) prevalence of intestinal parasitic infections in asymptomatic primary school children and this result might be because of the study sites are found in the similar geographical location and socioeconomic activities of the people(Table 2) and this finding was incomparable with the results obtained in a study conducted in different communities of different study sites among the school children in Nirobi [76] and in Ghana[77]. These variations in the prevalence of IPIs could be due to differences in geographical location and socioeconomic activities of the people, low level of awareness about the transmission of intestinal parasites and ignorance of health-promotion practices, the level of environmental sanitation and source of drinking water [78, 64, and 69].

Moreover, responses of the asymptomatic children on the associated predisposing factors related to parasitic infection showed mixed responses (Table 5) including majority of the respondents 225(53.3\%) got drinking water from pipe and $197(46.7 \%)$ respondents from unprotected well and river and 303 (71.8\%) did not dispose household wastes properly and $119(28.2 \%)$ dispose properly. Of the total participants, 181(42.9\%) did not wash their hands after defecation and 192(45.5\%) participants had frequent river water contact. Moreover, 186 (44.1\%) did not wash their hands before meal and. among the study participants, $157(37.2 \%)$ did not use shoe wearing consistently. Furthermore, among the study 
participants, 263(62.1\%), used to eat raw vegetables and unwashed fruits and 261(61.8\%) participants did not have cleaned and trimmed finger nails during data collection, disposing of wastes to environment (119\%).

Present study showed predisposing factors and this result was in line with this different studies which show predisposing actors such as drinking water from unprotected well and river, improper disposal of household wastes, air defecation due to improper use of latrine, not washing hands before meal, not wearing shoes consistently, not trimming finger nails regularly and habit of eating raw vegetables and unwashed fruits which are predisposing factors that are closely associated with intestinal parasitic infections[79,23,73,54,69].

In agreement with the present finding, another studies from Saudi Arabia show similar association of intestinal parasitic infections related to not properly hand washing habit of students[79] and this association was also reported from Ethiopia[59,71]. Moreover, in agreement with the present study, other similar studies also show students with untrimmed and unclean finger nail are more infected by intestinal parasitic infections as compared with students who had trimmed and cleaned their finger nails [52, 80, and 79]. Similarly, the likely hood of being infected by intestinal parasites was more among students who drink well water as compared with students who drink pipe water [81, 66, and 71]. Moreover, in agreement with present study, students who eat raw vegetables and unwashed fruit are more infected than their counter parts $[69,75]$ and, students who do not wash hands after defecation and students who do not use latrine always are more susceptible to parasitic infection than their counter parts[66,60].

Furthermore, the likely hood of being infected by hookworm is increased among students who do not use protective shoe as compared with students regularly use protective shoe [59, 52, 71, 31, 70].

\section{Conclusion}

The present study revealed that there was high prevalence of intestinal parasitic infection among the students in the targeted primary schools in Finote selam town. Intestinal parasite species diagnosed in the present study were G.lamblia, E. histolytica Hookworm, A.lumbricoides, T. trichiura, S. mansoni and H. nana with the highest prevalence of G. lamblia $(23.9 \%)$ and the lowest prevalence of $H$. nana $(0.2 \%)$. The study had also identified a number predisposing factors to intestinal parasitic infections like consumption of raw vegetables and unwashed fruits, not washing hands before meal and after toilet use, uncleaned and untrimmed finger nails, accessibility of drinking water source, defecation habit, consistency of river water contact and shoe wearing habit.

\section{Recommendations}

Based on the result of the study, the following interventions were recommended:

- Provision of health education by the health service units to improve hand washing practices using soaps before meals and after defecation and the level of environmental hygiene by avoiding open air defecation.

- There should be adequate provision of sanitary facilities in schools and in the communities focusing on water sanitation and hygiene initiatives.

- The local head masters should give attention to construction of public toilets for every household to avoid open defecation of the local people.

- School based deworming programs should be done regularly to control morbidity due to IPIs infections.

- Inspection should be conducted on school children by school head teachers and unit leaders for personal hygienic practices and shoe wearing habits.

- In order to obtain maximum benefits, further epidemiological studies focusing on control of parasitic infections and diseases should be undertaken in an integrated and coordinated manner. 


\section{Compliance with ethical standards}

\section{Acknowledgments}

We would like to thank Arba Minch University, for facilitating ground to this research work in general, in particular for financing the research activity. We are also grateful to Finoteselam Hospital and Health office of Finoteselam town for providing necessary information allowing the laboratory facilities and technical support of parasitological examination and all other individuals who contributed their share to the success of this study.

\section{Disclosure of conflict of interest}

The authors have not declared any conflict of interests.

\section{References}

[1] Saki J, Shahram K, Kambiz M, Mahmood C. Prevalence of intestinal parasitic infections among food handlers in Khuzestan, South West of Iran: A 10- year's retrospective study. African Journal of Microbiology Research. 2012; 6(10): 2475- 2480.

[2] WHO: Conquering, Suffering and Enriching Humanity Report of an Informal Consultation. 2000.

[3] Wakid HM. Improvement of Ritchie Technique by Identifying the Food That Can Be Consumed: Pre-analysis. J. App. Sci. Res. 2009; 5: 293-296.

[4] Mohammed K, Abdullah M, Omar J. Intestinal parasitic infection and assessment of risk factors in North-western Nigeria: A Community Based Study. 2015; 4(2): 141-5.

[5] Arora DR, Arora BB. Medical Parasitology, 3rd Edition. CBS Publishers and Distributors PVT Limited, New Delhi, India. 2011.

[6] Gamboa MI, Navone GT, Orden AB, Torres MF, Castro LE, Oyhenart EE. Socio environmental conditions, intestinal parasitic infections and nutritional status in children from a sub-urban neighborhood of La Plata: Argentina Eur. J Epidemiology. 2009; 14(1): 55-61.

[7] Niyyati M, Rezaeian M, Zahabion F. A survey on intestinal parasitic infections in patients referred to Hospital in Tehran. Pakistan J. Med Sci. 2009; 25(1): 87-90.

[8] Masoumeh R, Farideh T, Heshmatollah T. Intestinal parasitic infection among school children in Golestan Province; Iran Pak. J. Biol. Sci. 2012; 15: 19-25.

[9] Dib HH, Lu SO, Wen SF. (2008). Prevalence of Giardia lamblia with orwithout diarrhea in South East Asia and the Far East. Parasol Res. 2008; 103: 239-51.

[10] Ayeh-Kumi PF, Quarcoo S, Kwakye-Nuako G, Kretchy JP, Osafo-Kantanka. A. Prevalence of intestinal parasitic infections among food vendors in Accra Ghana. J. Trop Med Parasitol. 2009; 32: 1-8.

[11] Olubunmi O. Parasites of Man and Animals: Concept publication limited. 2013.

[12] Vandenberg O, Van Laethem Y, Souayah H, Kutane WT, Van Gool T. Improvement of routine diagnosis of intestinal parasites with multiple sampling and SAF-fixative in the triple-feces-test. Gastroenterology Belg. 2006; 69: 361-366.

[13] Mehraj V, Hatcher J, Akhtar S, Rafique G, Beg A. Prevalence and Factors Associated with Intestinal Parasitic Infection among Children in an Urban Slum of Karachi. PLoS One. 2008; 3(11): e3680.

[14] Ahmed T, Khanum H, Uddin MS, Barua P, Arju T, Kabir M, Haque R. Entameoba Histolytica, Giardia Lamblia and Cryptosporidium spp. infection in children in an urban slum area of Bangladesh Bio res. Comm. 2016; 2: 175-181.

[15] WHO. Eliminating Soil Transmitted Helminthiasis as a Public Health Problem in Children; Progress Report 20012010 and Strategic Plan 2011-2020, Switzerland.2012.

[16] Rice J, Skull S, Pearce C, Mulholl N, Davie G, Carapetis J. Screening for intestinal parasites in recently arrived children from East Africa. Journal of pediatrics and child health. 2003; 39(6): 456-9.

[17] Gimba U, Dawam N. Epidemiological status of intestinal parasitic infection rates in children attending Gwagwalada township clinic, FCT-Abuja, Nigeria. Am J. Res. Commune. 2015; 3(2): 97- 11. 
[18] Aschalew Gelaw, Belay Anagaw, Bethel Nigussie, Baye Gelaw. Prevalence of intestinal parasitic infections and risk factors among school children at the University, Gondar Community School, Northwest Ethiopia. Public Health. 2013; 13(304): 1471-2458.

[19] Amare Mengistu, Solomon Gebre -Silassie, Tesfaye Kassa. Prevalence of intestinal parasitic infections among urban dwellers in south west Ethiopia. J. Health and Dev. 2007; 21(1): 12-7.

[20] Tadesse Dejene, Tsehaye Asmelash. Impacts of irrigation on the prevalence of intestinal parasitic infections. Ethiop J. Health Dev. 2008; 18(2): 13-36.

[21] Sayyari A, Imanzadeh F, Bagheri A, Karami H. Prevalence of intestinal parasitic infections in the Islamic Republic of Iran. Health J. 2005; 11: 377- 383.

[22] Ostan I, Kilimcioglu AA, Girginkardesler N, Ozyurt BC, Limoncus ME, Ok UZ. Health inequities: Lower socioeconomic conditions and higher incidences of intestinal parasites. Public Health. 2007; 7(342): 1-8.

[23] Alemayehu Getachew. Prevalence and Intensity of Intestinal Parasitic Infections and Associated Risk Factors among Households around Akaki River and Aba Samuel Dam, Addis Ababa, Ethiopia.; SGSHU. 2014; 1(1): 1-50.

[24] Brooker S, Hotez PJ, Bundy DA. Hookworm-Related Anemia among Pregnant Women: A Systematic Review. Trop. Dis. 2008; 2: 291.

[25] Daryani A, Sharif M, Nasrolahei M, Khalilian A, Mohammadi A, Barzegar G. Epidemiological survey of the prevalence of intestinal parasites among school children in northern Iran: Trans, R. Soc. Trop. Med. Hyg. 2012; 106(8): 455-459.

[26] Cooper NR, Clin, Corachan M. Complement evasion strategies of Schistosomiasis and international travel. Immun. Today. 2007; 35: 446-50.

[27] Taheri F, Namakin K, Zarban A, Sharifzadeh G. Intestinal parasitic infection among school children in South Khorasan Province, Iran. J. Res Health. 2011; 3(1): 45-50.

[28] Muhajir A, Hajissa K, Mohamed Z, Abdel-Aal A. Prevalence of intestinal parasitic infection among children in alkalakla, Khartoum. Sudan World App Sci. J. 2017; 35(2): 219-22.

[29] Kumie Abera, Ali Ahmed. An overview of environmental health status in Ethiopia with particular emphasis on drinking water and sanitation: A literature survey. Ethiop J. Health Dev. 2005; 19(2): 36-42.

[30] WHO: Diarrhea, Why children are still dying and what can be done? 2009.

[31] Melesse Birmeka, Kelebesa Urga, Beyene Petros. Prevalence and determinants factors of intestinal parasitic infections among the primary school children in Gurage zone, south central Ethiopia. Microbiology. 2017; 82 (20): 59-70.

[32] Sackey ME, Weigel MM, Armijos RX. Predictors and nutritional consequences of intestinal parasitic infections in rural Ecuadorian children. J. Trop Pedant. 2003; 49: 17-23.

[33] Rodriguez-Morales AJ, Barbella RA, Case C, Arria M, Ravelo M, Perez H, Urdaneta O, Gervasio G. Intestinal parasitic infections among pregnant women in Venezuela: Infect Dis Obstetric Gynecol. 2006; 23: 125.

[34] Hotez PJ, Alvarado M, Basanez M-G, Bolliger I, Bourne R, Boussinesq M. A study on the global burden of a disease: Interpretation and implications for the neglected tropical diseases. PLoS Negl Trop Dis. 2014; 8(7): 203-308.

[35] Abayneh Unasho. Infections of Common Protozoan and Helminthe Parasites in the Intestine, under finger nail's content and associated predisposing factors among the asymptomatic food handlers working in the student cafeteria of Dilla University. International Journal of Medical and Health Science. 2019; 5(2): 11- 28.

[36] Netsanet Worku, Berhanu Erko, Workineh Torben, Mulugeta Belay, Afework Kassu, Teshome Fetene. Malnutrition and intestinal parasitic infections in school children of Gondar, Northwest Ethiopia. Ethiopian Med J. 2009; 47(1): 9-16.

[37] Shrestha A, Narayan KC, Sharma R. Prevalence of intestinal parasitosis among school children in Baglung districts of Western Nepal. Univ. Med J. 2012; 10: 3-6.

[38] Kloos H. Human behavior, health education and schistosomiasis control. A review. Soc. Sci. Med. 2005; 40: 14971511.

[39] Alwabr G, Al-Moayed E. Prevalence of intestinal parasitic infections among school children of al-Mahweet governorate; Yemen. Eur. J. Biol. Res. 2016; 6(2): 64-73. 
[40] Finoteselam city administration, West Gojjam zone Amhara region, Ethiopia. 2018.

[41] Finoteselam city administration Geographical Information System Map of Ethiopia Showing Amhara Region and Fitnoteselam town. 2019.

[42] Charan J, Biswas T. How to Calculate Sample Size for Different Study Designs in Medical Research. Indian Journal of Psychological Medicine. 2013; 35(2): 121-126.

[43] WHO: Prevention and control of schistosomiasis and soil-transmitted helminthiasis. 2004.

[44] WHO: Urges governments to increase investment to tackle neglected tropical diseases. 2015.

[45] Garcia LS, Arrowood MN, Visvesvara G. Laboratory diagnosis of parasites from the gastrointestinal tract. Clin Microbiol Rev. 2018; 31: 25-17.

[46] Abraraw Abate, Biniam Kibret, Eylachew Bekalu, Sendeku Abera, Takele Teklu, Aregawi Yalew, Mengistu Endris, Ligabaw Worku, Zinaye Tekeste. Cross Sectional Study on the Prevalence of Intestinal Parasites and Associated Risk Factors in Teda Health Centre, Northwest Ethiopia. ISRN. Parasitology. 2013; 5: 5402-5745.

[47] Biniam Mathewos, Abebe Alemu, Desalegn Woldeyohannes, Agersew Alemu, Zelalem Addis. Current status of soil transmitted helminthes and Shistosoma mansoni infection among children in two primary schools in north Gonder northwest Ethiopian: a cross sectional study. Res. Notes. 2014; 7(88): 56-63.

[48] Eleni Kidane, Sissay Mekir, Amha Kebede, Mulugeta Desta. Prevalence of Intestinal Infections and Associations with Anthropometric measurements of school children in selected primary schools, Wukro Town eastern Tigry Ethiopia. Int. J. curr. Microbiology: App. Sci. 2014; 3(3): 11-29.

[49] Mengistu Wale, Melaku Wale, Tesfu Feknesa. The prevalence of intestinal helminthic infections and associated risk factors among school children in Lumamae town, north-West Ethiopia. JPVB. 2014; 3(5): 75-81.

[50] Araya Gebreyesus, Mekonen Teferi, Letemichal Negash, Dejen Yemane, Haftu Temesgen, Tsehaye Asmelash. Intestinal parasitosis, anaemia and risk factors among pre-school children in Tigray Region, Northern Ethiopia. 2019.

[51] Fatni C, Fatni H, Romero D, Olmo F, Rosales M. Intestinal parasitism in Moroccan children comparative quantitative study of the Faust's and Ritchie's coprological methods. 2014; 11(1): 53-64.

[52] Desta Haftu, Negussie Deyessa, Eskzyiaw Agedew. Prevalence and determinant factors of intestinal parasites among school children in Arba Minch town, southern Ethiopia. Am. J. Public Health Res. 2014); 2(5): $247-54$.

[53] Admasu Haile, Temesgen Abera, Daniel Dana. The Prevalence of Intestinal Parasitic Infection and Associated Factors among Primary School Children in Gurage Zone, South Ethiopia. An International Peer-reviewed Journal. 2017; 15: 2222-5668.

[54] Birhanu Megerssa. Prevalence of intestinal parasitic infection among Libbefana Kindergarten Children in Kirkos Sub city, Addis Ababa, Ethiopia. Infect. Disease. 2018; 1: 1-46.

[55] Wiser MF. Intestinal Protozoa Tulane University Last update on June 3, 2015.

[56] Danladi, Nigeria: Prevalence of gastrointestinal parasites among primary school children in bosso local government area of niger state, Nigeria. 2015.

[57] Wafa A, I.AL-M. The Prevalence of Intestinal Parasites and Risk Factors among Preschool children in Riyadh, Saudi Arabia.Journal of parasitology. 2015; 10(1): 31-41.

[58] Bahmani P, Mleki A, Sadeghi S, Shamoradi B. Prevalence of intestinal protozoan infections and associated risk factors among schoolchildren in Sanandaj City: Iran. J. Parasitology. 2017; 12(1): 108-16.

[59] Mulat Alamir, Worku Awoke, Amisalu Feleke. Intestinal parasitic infection and associated factors among school children in Dagi primary school, Amhara National Regional State, Ethiopia. Health. 2013; 5(10): 1697-701.

[60] Tilahun Workneh, Ahmed Esmael, Mekonen Ayichiluhm. Prevalence of intestinal parasitic infections and associated factors among Debre Elias primary school, north-West Ethiopia. J Bacteriol Parasitol. 2014; 5(181): 11-9.

[61] Asnakech Deneke. Intestinal Parasitic Infections among Patients Visiting Gorebella Health Center, North-Central Ethiopia. Int.Gastro logy. 2016; 5: 26-45. 
[62] Erismann S, Diagbouga S, Odermatt P, Knoblauch A, Gerold J, Shrestha A, Grissoum T, Kaboré A. Prevalence of intestinal parasitic infections and associated risk factors among school children in the plateau Central and Centre-Ouest regions of Burkina Faso. Parasite Vectors. 2016; 9: 554.

[63] Hussein Sharif, Imad Abdelgalil, Mosab Nouraldein, Ahmed Mustafa Bashir. Prevalence of Intestinal Parasites among Selected Group of Primary School Children in Alhag Yousif Area, Khartoum, Sudan. International Journal of Medical Research \& Health Sciences. 2017; 6(8): 125-131.

[64] Fiseha Wadilo, Fithamlak Solomon, Amsalu Arota, Yishak Abraham. Intestinal Parasitic infection and Associated Factors among Food Handlers in South Ethiopia. Journal of Pharmacy and Alternative Medicine. 2016; 12, 22225668.

[65] Ashenafi Abossie, Mohamed Seid. Assessment of the prevalence of intestinal parasitosis and associated risk factors among primary school children in Chencha town, Southern Ethiopia. Public Health. 2014; 14(166): 14712458.

[66] Asrat Ayalew, Tewodros Debebe, Alemayehu Worku. Prevalence and risk factors of intestinal parasites among Delgi school children, North Gondar, Ethiopia. 2011; J. Parasitology Vector Biol. 3(5): 75-81.

[67] Emile N, Bosco N, Karine B. Prevalence of intestinal parasitic infections and associated risk factors among Kigali Institute of Education students in Kigali.Rwanda. Tropical Biomed. 2013; 30(4): 718-26.

[68] Gebremichael Gebretsedik. Prevalence of intestinal parasite and associated risk factors among school children of Homesha District in Benishagul-Gumuz regional state western Ethiopian. J. Fam. Med Health Care. 2016; 2 (4): 57-64.

[69] Baye Sitotaw, Haileyesus Mekuriaw, Destaw Damtie. Prevalence of intestinal parasitic infections and associated risk factors among Jawi primary school children, Jawi town, northwest Ethiopia. 2019; Infectious Diseases.19: 341.

[70] Getaneh Alemu, Zeleke Aschalew, Eshetu Zerihun. Burden of intestinal helminthes and associated factors in three years after initiation of mass drug administration in Arbaminch Zuria district, Southern Ethiopia. Infectious Diseases. 2018; 18: 435-445.

[71] Mulusew Andualem. Parasitic Infection and Associated Factors among the Primary School Children in Motta Town, Western Amhara, Ethiopia. American Journal of Public Health Research. 2014; 2(6): 248-254.

[72] Alganesh Gebreyohanns, Melese Hailu,Mistire Wolde, Gemechu Leta Geremew Tasew. Prevalence of intestinal parasites versus knowledge, attitude and practices with special emphasis on Schistosoma mansoni among individuals who have river water contact in Addiremets town, Western Tigray, Ethiopia. PLoS ONE. 2018; 13(9): e0204259.

[73] Tamirat Hailegebriel. Prevalence of intestinal parasitic infection and associated risk factors among students at Dona Berber primary school, Bahir Dar, Ethiopia. Infectious Disease.2017; 17(362): 1-8.

[74] Berhanu Erko, Girmay Medhin. Human Helminthiasis in Wondo-Genet, Southern Ethiopia, with emphasis on geohelminthiasis. Ethiop. Med. J. 2003; 41: 333-334.

[75] Megbaru Alemu, Abay Anley, Kiros Tedla. Magnitude of Intestinal Parasitosis and Associated Factors in Rural School Children, North west Ethiopia. Ethiop J. Health Sci. 2019; 29(1): 923-935.

[76] Benedict $M$. The influence of intestinal parasites on academic performance among primary school children in Nairobi province, Kenya. Infect. Disease. 2010; 1: 1-54.

[77] Philip T. A Comparative Study of Intestinal Parasitic Infection and Associated Risk Factors among Primary School Children in Six Neighboring Communities in Kumasi, Ghana. 2012.

[78] Vandemark LM, et al. Social science implications for control of helminthes infections in Southeast Asia. Advances in Parasitology. 2010; 73(20): 137-170.

[79] Al-Mohammed H, Amin T, Aboulmagd E, Hablus H, Zaza B. Prevalence of intestinal parasitic infections and its relationship with socio-demographics and hygienic habits among male primary schoolchildren in Al-Ahsa, Saudi Arabia. Asian Pac. J. Trop Med. 2010; 32: 906-12.

[80] Berhanu Elfu. Nutritional Status and Intestinal Parasitic infection in School Age Children: A Comparative CrossSectional Study. Int. J. Pediatr. 2016; 1: 621-808. 
World Journal of Advanced Research and Reviews, 2020, 08(02), 173-188

[81] Abebe Alemu, Asmamaw Atnafu, Zelalem Addis, Yitayal Shiferaw, Takele Teklu, Biniam Mathewos, Wubet Birhan, Simon Gebretsadik, Baye Gelaw. Soil transmitted helminthes and Schistosoma mansoni infections among school children in zarima town, northwest Ethiopia. 2011; 11(189): 1-7. 